Relations industrielles

Industrial Relations

\title{
The Rule of Law in European Integration, Stuart A. Scheingold, McGill University Press, Montréal, 1965. 331 pages.
}

\section{Ronald Pleau}

Volume 21, numéro 2, 1966

URI : https://id.erudit.org/iderudit/027685ar

DOI : https://doi.org/10.7202/027685ar

Aller au sommaire du numéro

Éditeur(s)

Département des relations industrielles de l'Université Laval

ISSN

0034-379X (imprimé)

1703-8138 (numérique)

Découvrir la revue

Citer ce compte rendu

Pleau, R. (1966). Compte rendu de [The Rule of Law in European Integration, Stuart A. Scheingold, McGill University Press, Montréal, 1965. 331 pages.] Relations industrielles / Industrial Relations, 21(2), 302-302.

https://doi.org/10.7202/027685ar

Tous droits réservés (C) Département des relations industrielles de l'Université Laval, 1966
Ce document est protégé par la loi sur le droit d'auteur. L’utilisation des services d'Érudit (y compris la reproduction) est assujettie à sa politique d'utilisation que vous pouvez consulter en ligne.

https://apropos.erudit.org/fr/usagers/politique-dutilisation/ 
Jusqu'ici le législateur donne une protection au brevet d'invention, mais non au secret technique. Et si un tel système a pu être établi pour le premier, on peut aussi le faire pour le second, en établissant un document de référence (attestant la possession personnelle) en termes de brevet.

\section{Fernand Blais}

The Rule of Law in European Integration, Stuart A. Scheingold, McGill University Press, Montréal, 1965. 331 pages.

Parmi les institutions dont nous entendons le plus parler dans l'économie européenne moderne, nous remarquons la Communauté du charbon et de l'acier (le plan Schuman), le Marché commun et l'Euratom. Or, pour que les états membres de ces institutions qui se sentent lésés dans leurs droits et qui ont des griefs à formuler à l'endroit des systèmes puissent avoir certains recours, il a fallu instituer également une cour de justice pour l'audition de ces cos.

Ainsi dans cet ouvrage l'auteur examine le rôle de cette cour et établit la croissance de son approche fonctionnelle vis-à-vis les cas économico-juridiques qui lui sont présentés. II s'en tient ici à ceux formulés dans le cadre du plan Schuman lesquels sont d'ailleurs semblables aux autres et à ceux de l'intégration régionale en général. Ces cas constituent en quelque sorte une histoire judiciaire de cette institution et fournissent un excellent terrain d'étude pour une analyse.

Scheingold essaie de voir jusqu'à quel point après dix ans d'application du système de la communauté cette cour de justice s'est ajustée avec succès à son environnement et si cette dernière a apporté une contribution véritable au succès des Communautés mentionnées.

Selon les termes de l'auteur lui-même le but de cet exposé est de renseigner sur la relation réciproque qui existe entre les aspects légal et politique de l'intégration européenne.

Les deux premiers chapitres de cette étude soulignent la tension qui existe entre les rôles politiques et judiciaires de la cour de justice. Les chapitres suivants serviront à justifier l'argument qui veut que la cour oit adopté des techniques fonctionnelles et à démontrer le succès des méthodes fonctionnelles pour imposer des règles normatives au processus d'intégration.
Mais pour comprendre complètement la contribution du fonctionalisme, l'auteur voit la nécessité d'un côté, d'explorer ses limitations et, d'un autre côté, d'établir la faiblesse des méthodes olternatives adoptées par cette cour. C'est à cette tâche qu'il se consacre dans les deux chapitres suivants de la partie II.

Lo partie III traitera de l'évolution de la technique fonctionnelle et les IVe et $\mathrm{Ve}$ parties, de ses limitations, et plus spécialement des problèmes d'intégration partielle et de changement politique. II termine enfin sur une série de conclusions des plus intéressantes.

Le tout constitue donc une analyse des problèmes de conflits entre la recherche de la stabilité et le processus dynamique de I'intégration et de l'écart entre les institutions nationales juridiques et politiques.

\section{Ronald Pleau}

The Family, William J. Goode, Foundations of Modern Sociology Series, Prentice-Hall, Inc., Englewood Cliffs, New Jersey. 1965. 120 pages

II sera de tout temps nécessaire de traiter des relations familiales et d'insister sur les résultats et découvertes de la théorie sociologique à ce sujet.

Ainsi par exemple en étudiant les relations entre les systèmes de famille et la structure sociale plus vaste, nous pouvons dire que nous ne devons plus considérer la famille comme un simple ensemble de variables dépendantes expliquées par les \& forces économiques » seules. II faut chercher les relations entre les variables centrales et s'efforcer d'explorer la structure sociale plus vaste.

Quant au problème des valeurs, nous pouvons dire que l'analyse que contient ce volume n'en fait pas une évaluation mais que la sociologie comme science peut examiner de temps en temps certains modes alternatifs de vivre.

L'cuteur divise son oeurre en dix courts chapitres et considère la famille et les systèmes de relations familiales sous des aspects très variés bien que très loggiquement relics entre eux.

Toutes les sociétés ne conçoivent pas la famille de la même façon: certaines ont même des préjugés ou sujet de cette dernière. Mais quoiqu'il en soit, la famille restera toujours une institution unique qui 